\title{
Eliminating Forgers Based on Intra Trial Variability in Online Signature Verification Using Handglove and Photometric Signals
}

\author{
Andrews Samraj $^{1}$, Shohel Sayeed ${ }^{1}$, Loo Chu Kiong ${ }^{1}$, Nikos E. Mastorokis ${ }^{2}$ \\ ${ }^{1}$ Faculty of Information Science and Technology, Multimedia University, Malacca, Malaysia \\ ${ }^{2}$ Faculty of Engineering, Industrial Engineering Department, Technical University of Sofia, Sofia, Bulgaria \\ E-mail: andrews.samraj@mmu.edu.my
}

Received March 5, 2010; revised June 9, 2010; accepted July 15, 2010

\begin{abstract}
The novel reinforcement to the data glove based dynamic signature verification system, using the Photometric measurement values collected simultaneously from photo plethysmography (PPG) during the signing process is the emerging technology. Skilled forgers try to attempt the genuine signatures in many numbers of trials. The wide gap in the Euclidian distances between forgers and the genuine template features prohibits them from successful forging. This has been proved by our repeated experiments on various subjects using the above combinational features. In addition the intra trial features captured during the forge attempts also differs widely in the case of forgers and are not consistent that of a genuine signature. This is caused by the pulse characteristics and degree of bilateral hand dimensional similarity, and the degrees of pulse delay. Since this economical and simple optical-based technology is offering an improved biometric security, it is essential to look for other reinforcements such the variability factor considerations which we proved of worth considering.
\end{abstract}

Keywords: Photo Plethysmography, Data Glove, Signature Verification, Multimodal Biometrics

\section{Introduction}

Enhancements to the signature verification systems has been suggested by many researchers [1-3] and bio signal based security features are also considered as a unique alternatives for applications that require some document evidence like signing cheques and security documents. The signal based biometrics in the only applicable means for people with physical disability [4].

Making use of multimodal biometric technology which provides unique and robust identification features for every individual is in great demand in security environments that require high quality authentication methods. Using PPG wave forms to distinguish individuals using their biometric component was suggested by researchers in 2007 [5] and is employed in protected applications like e-transactions and access control mechanisms.

As a fortification to the current signal based dynamic signature verification system, we have used a new method by using the combination of the plethysmographic component along with the data glove signals to make the authentication process more robust and distinctive.

The possibility of skilled forging is reduced by the PPG feature that brings in the hand and heart dimensions of an individual into the signature feature vector. In order to further reinforce the effectiveness of the system here in this research work we have considered the intra trial variation approach to further validate the signature process. This method assures the elimination of skilled forging by a multi level authentication.

\section{The Equipment}

The plethysmographic system, a simple equipment that functions on the intensity of light reflected from the skin's surface. The red cells count below the skin is considered to determine the volume of blood in the particular area. The recorded signal posses the measurement of changes in venous blood volume and the arterial blood pulsation in the arterioles, hence representing the heart rate. There are two values supplied by the system and are the measurements of transmission and reflectance. A sam- 
ple signal produced by the PPG is shown in Figure 1.

Similarly, the data glove is used for dynamic signature verification and that is easy to use, free from image and material of signature medium as well as no scanning processes is required. It involves only a direct acquisition of signals from the subjects while they write down their signatures, preprocess it, extract the feature, match it to classify and decision making. The data glove offers the users comfort, ease of application, and it comes with a small form factor with multiple application drivers, high data quality, low cross-correlation and high frequency data lodging. It measures finger flexure (2 sensors per finger) as well as the abduction between fingers. The system interfaces with the computer via a cable to the USB port (Platform Independent). It features an auto calibration function, 8-bit flexure and abduction resolution, extreme comfort, low drift and an open architecture. It can also be operated wirelessly to interface with the computer via Bluetooth technology up to $20 \mathrm{~m}$ distance.

Similarly, the data glove is used for dynamic signature verification and that is easy to use, free from image and material of signature medium as well as no scanning processes is required. It involves only a direct acquisition of signals from the subjects while they write down their signatures, preprocess it, extract the feature, match it to classify and decision making. The data glove offers the users comfort, ease of application, and it comes with a small form factor with multiple application drivers, high data quality, low cross-correlation and high frequency data lodging. It measures finger flexure (2 sensors per finger) as well as the abduction between fingers. The system interfaces with the computer via a cable to the USB port (Platform Independent). It features an auto calibration function, 8-bit flexure and abduction resolution, extreme comfort, low drift and an open architecture. It can also be operated wirelessly to interface with the computer via Bluetooth technology up to $20 \mathrm{~m}$ distance.

One glove fits many hands since it is made-up of stretchable material "A".

The data Glove and the signature verification process using the glove is shown in Figure 2. The output of the probe is fed into the serial port of a pulse oximetry module (from Dolphin Medical, Inc.) Measurements were taken for 50 signatures from 14 sensors of the data glove, and four led's of plethysmogram fixed on the subject as seen in Figure 3.

\section{The Fusion of Photo Plethysmography System with Data Glove Signals}

\subsection{Subjects and Signal Acquisition Methods}

In this study, the data glove signals and photo plethysmographic signals were recorded from 6 volunteered

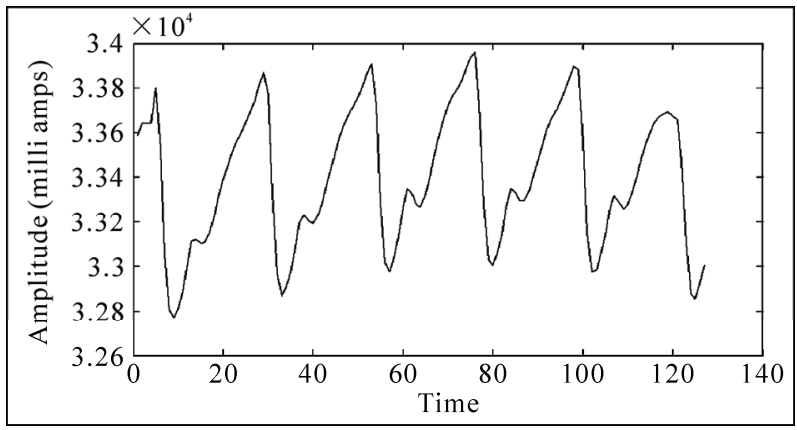

Figure 1. The PPG signal pattern during one signature.

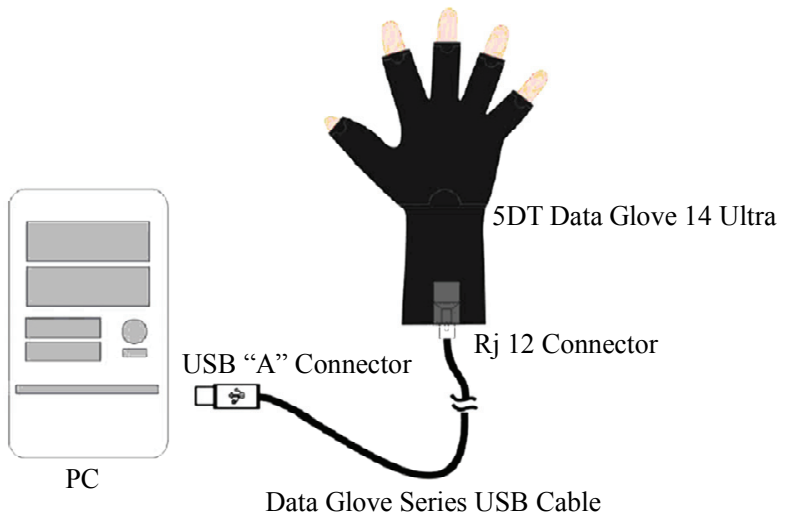

Figure 2. The data glove.

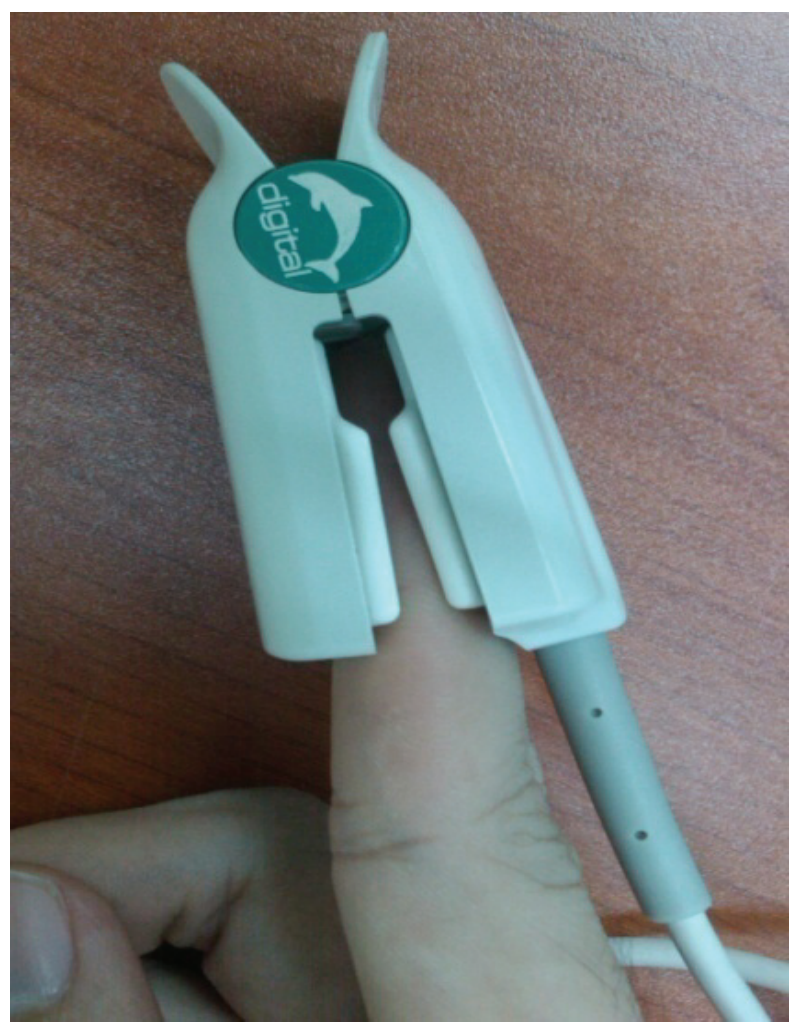

Figure 3. The plethysmogram. 
subjects. Two subjects were considered as original signers and other four were the skilled forgers. The data glove signals and the peripheral volume pulses (PPG) were sampled at $61 \mathrm{~Hz}$. Both the signals were recorded from the subjects simultaneously while they were signing.

The subjects were selected among our co-researchers and the average age of the subjects is 34 .

The dynamic features of the data glove signal comprises of

1) Distinctive patterns to an individual's signature,

2) The hand dimensions,

3) Time taken to complete a signature process,

4) Hand trajectory dependent rolling.

These factors contributes to the feature of the signal captured from the data glove and make it more suitable to trust for use in signature verification since it provides data on the dynamics of pen movement and the individual's hand dimensions. Along with these four components that represent a person's identity, the heart rate reflected by the plethysmographic signals is also measured as the fifth component to reinforce the system's distinctiveness. The photometric signal consists on the volume of blood that flows through the blood vessels per pulse during every beat of the heart.

\subsection{Experimental Setup}

The recordings of signals are arranged in such a way that one of the subject writing original signatures was allowed to sign 50 original (own) signatures and the subjects who are assigned to forge are allowed to observe it to do the skilled forgery [6].

The forgers are given generous exercise to forge against the two genuine signatures by giving special sessions to practice the original signatures. The subjects are seated in a comfortable chair located in a sound protected room. The data glove was fixed on their right hand and the photoelectric probe was fixed to the index finger of their left hand.

All the subjects appointed for forging were allowed to sign 50 forge signatures one by one each with the help of a tracing paper placed on the original signatures after successful training. The subjects were asked to write the signatures, in two sessions, with an interval of 24 hours. Fifty signatures were collected per subject in one session. The skilled forging of the original signatures from forging subjects were also collected in the same intervals. Forging with 50 signatures per original subject takes a total of 100 signatures per session for two original signatures.

The PPG signal during the signing in process was also recorded for every subject from all the 14 electrodes embedded in the data glove.

Hence there were 200 original signatures and 800 forgings were recorded and considered for analysis. Similarly 1000 simultaneous PPG recordings were also included in the analysis.

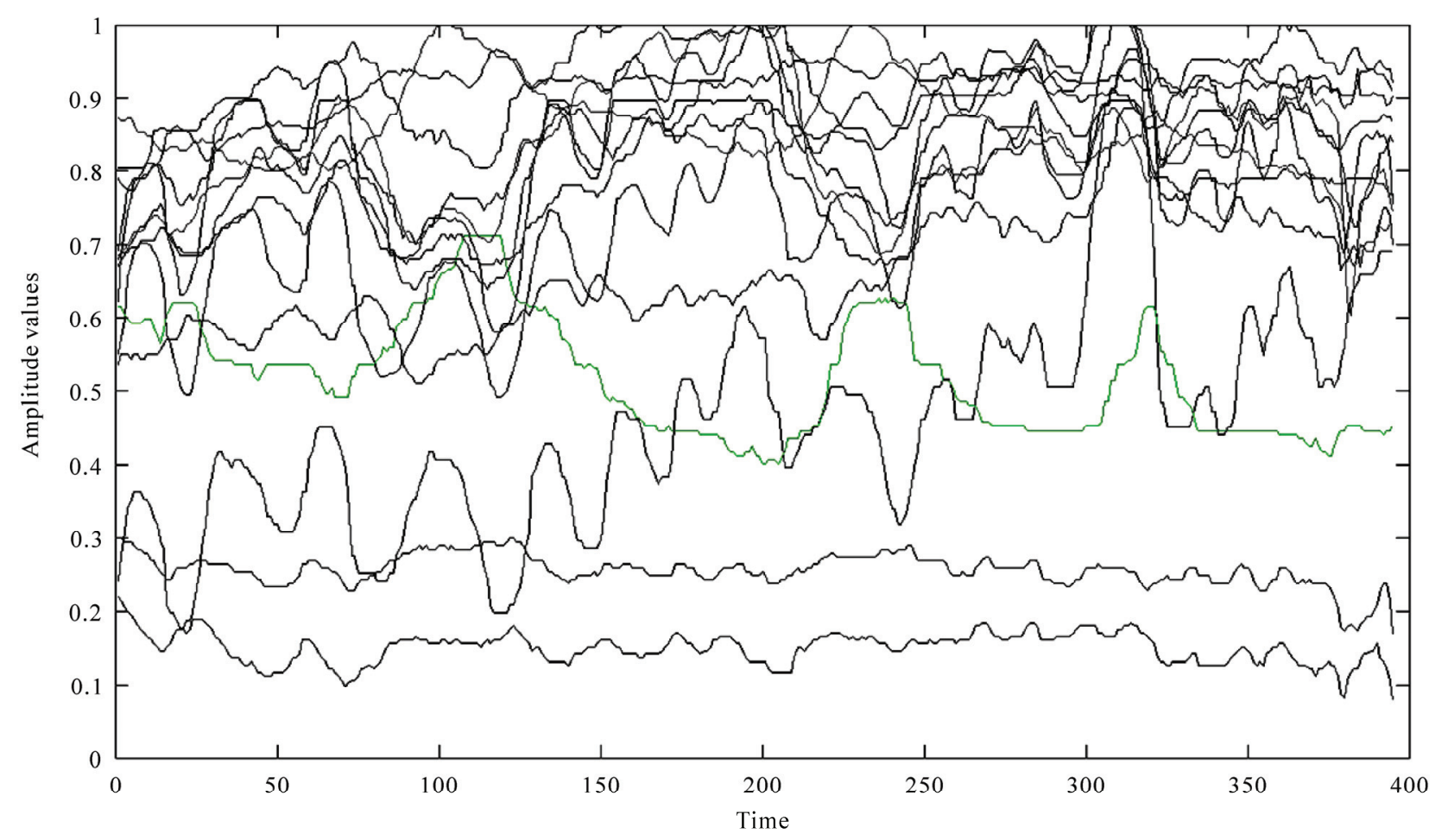

Figure 4. The data glove signal pattern during one signature. 


\subsection{Preprocessing and Feature Vector Construction}

The dimension of each recordings of hand glove signal, $\boldsymbol{A}$ is of order $n$ by $m$, where $n$ is the number of electrodes and $m$ is the number of sequential samples per second. $n$ was fixed as 14 throughout the experiment, and $m$ differs in milliseconds as the intra and inter subject vary in signature timings. A sample of the plotted handglove signals are shown in Figure 4.

The dimension of every PPG, matrix $\boldsymbol{B}$ is $j$ by $k$, where $j$ is the number of LEDs and $k$ is the number of sequential samples in one second. Throughout the experiment, $j$ was fixed as 4 and $k$ was taken up to the exact time length of $m$.

To condense the dimension and to reduce the effects of overlapping spectral information between noise and signature features, singular value decomposition (SVD) approach was applied to both matrix $\boldsymbol{A}$ and $\boldsymbol{B}$.

Since there is a real factorization for any real $n X m$ matrices, The SVD of matrix $\boldsymbol{A} \& \boldsymbol{B}$ are is given by

$$
\begin{aligned}
& A=U . S . V^{T} \\
& B=R . F \cdot Q^{T}
\end{aligned}
$$

where $\boldsymbol{U}(\mathrm{m}$ by $\mathrm{m}), \boldsymbol{R}$ ( $j$ by $j) \boldsymbol{Q}(k$ by $k$ ) and $\boldsymbol{V}(n$ by $n)$

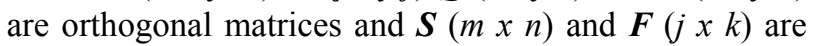
the diagonal matrices. The columns, $u_{i}$ and $v_{i}$ of $U$ and $V$ are the left and right singular vectors respectively, and the diagonal elements of $o_{i}^{*}$ of $\boldsymbol{S}$ are called the singular values. The columns, $r_{i}$ and $q_{i}$ of $\boldsymbol{R}$ and $\boldsymbol{Q}$ are the left and right singular vectors respectively, and the diagonal elements of $o_{i}$ of $\boldsymbol{S}$ are called the singular values.

Next, the singular values for each signal are arranged on the main diagonal in such an order:

$$
\sigma_{1} \geq \sigma_{2} \geq \sigma_{3} \cdots \geq \sigma_{r+1}=\cdots=\sigma_{p}=0
$$

The singular values calculated from the Matrix A are considered as the total Energy of matrix $A$. [7], and are measured in the direction of ith left singular vector of the matrix $A$.

$$
E[A]=\|A\|_{F}^{2}=\sum_{i=1}^{n} \sum_{j=1}^{m} a_{i j}^{2}
$$

Similarly through SVD, the diagonal entries $\sigma_{i}$ are the singular values of any matrix $A, A$ can be written as the sum of rank one matrices as $r=\operatorname{rank}(A)$.

$$
A=\sum_{i=1}^{r} \text { ui. } \sigma i . v_{i}^{T}
$$

where $\left(u_{i}, \sigma_{i}, v_{i}\right)$ is the ith singular triplet of matrix $A$.The oriented energy of matrix $A, E_{q}$ is measured in direction $q$ is delineated as

$$
E_{q}[A]=\sum_{K=1}^{n}\left(q^{T} \cdot a_{k}\right)^{2}
$$

In general the energy $E_{Q}$ measured in subspace $Q \in$ $R^{m}$ is given as

$$
E_{Q}[A]=\sum_{K=1}^{n}\left\|P_{Q}\left(a_{k}\right)\right\|^{2}
$$

The SVD can be related to the minima or maxima of the oriented energy distribution as follows.

$$
\begin{aligned}
& \max _{q \in U B} E_{q}[A]=E_{u 1}[A]=\sigma_{1}^{2} \\
& \min _{q \in U B} E_{q}[A]=E_{u n}[A]=\sigma_{n}^{2}
\end{aligned}
$$

From this it is proved that the oriented energy measured in the direction of the $i$ th left singular vector of the matrix $A$ is equal to the square of $i$ th singular value. Hence it is determined that the singular value decomposition protects the characteristics of the source signal matrix given by the $m$ signal samplings from $n$ electrodes.

Matrix $B$, used to incorporate PPG representation was also subjected to exactly similar SVD process to estimate the singular values for use in feature vector.

The average size of the glove signature matrix is $(14,234)$ as well as the average size of the PPG signal matrix is $(4,234)$. After the application of SVD the features are reduced to $(14,1)$ and $(4,1)$ respectively.

We were used the $l$-largest singular values of $\boldsymbol{A}$ as well as $q$-largest singular values of $\boldsymbol{B}$ as feature contents representing every single data glove signal and PPG respectively. Therefore, the entire signal $\boldsymbol{A}$ is now represented by a highly discriminate feature vector of length $\boldsymbol{A}(l)$ and the entire PPG is represented by $\boldsymbol{B}(q)$. These $l$ and $q$ largest singular value features of $\boldsymbol{A}$ and $\boldsymbol{B}$ contain the feature component of the subjects' unique signature ID that discriminates the original from forge signatures. To minimize computational complexity, we set the $l$ value to be five and $q$ to 2 throughout these experiments, subsequent to its superior performance during our preliminary simulations.

$$
\begin{aligned}
& F s=\left[A_{i}, \ldots A_{j}\right] \\
& F p=\left[B_{i}, \ldots B_{q}\right]
\end{aligned}
$$

where $i=1, J=5$ and $q=2$;

The fused feature

$$
F=\left[F_{s}, F_{p}\right]
$$

reflects the pattern of integrated signature components with the heart rate variability for further matching and classification.

\subsection{Matching and Classification}

The reference signature along with the reference PPG $F_{G}$ (Genuine Factor) was computed from a set of reference enrollment samples. The pair having minimal overall angle to the rest of signature, PPG pairs was selected as the reference signature to which all the comparisons where carried out. The genuineness of any factor pair $F_{i}$ 
is decided by the similarity factor (SF) to both the components of $F_{G} \& F_{i}$ are calculated as the angle between their principle subspaces.

\section{Results and Discussions}

Figure 5 gives Euclidean distance between the genuine reference signature PPG fusion factors with other genuine signature and forge signatures with the corresponding PPG of the subjects. The Euclidian distance is calculated using

$$
d=\sqrt{\sum_{j=1}^{L}\left(x_{j}-t_{j}\right)^{2}}
$$

Ten random sample distances across the two sessions were shown taken for considerations and other signatures were also giving similar results. These results were reported in our previous works [4].

The Equal Error Rate (EER) can be calculated if and only if a set of False Acceptance rate (FAR) and False Rejection Rate (FRR) are available. In this experiment, both are found to be zero and hence could not able to draw a curve of FAR and FRR to find the intersection point which is EER.

As an enhancement to this system we intended to find the consistency of the signatures written by the forgers with that of the consistency of the genuine signatories. This is to identify the best forger and later this factor may be used to enhance the authenticity of the entire system using the distinct quality of inter trial coherence.

Table 1 shows the results in terms of Euclidian distance between the signatures cum PPG fusion template to every subject's with their own signature cum PPG features found in different trials. We found that the consistency of the genuine signatory is consistence and all the other four skilled forgers were not able to retain their consistency across the trails. This can be seen from the zigzag lines from Figure 6.

The performance of the data glove declines with the reduction of sensors. The performance degradation with the reduction of electrode channels from the hand glove were reported in our previous works [8] to minimize the hardware and volume overheads in data processing. But the proposed technique of this paper helps to eliminate the said problem by providing strong reinforcements provided in two levels. The first one being the PPG factors and the second one is the intra trial variability. Table 1 shows the values of intra trial variability with the corresponding reference temples of every subject.

In all the ten trials the Euclidean distance is calculated against the templates of the individual subjects' training to write the same signature. The proposed signature and PPG combination is not altered through out the experiment.

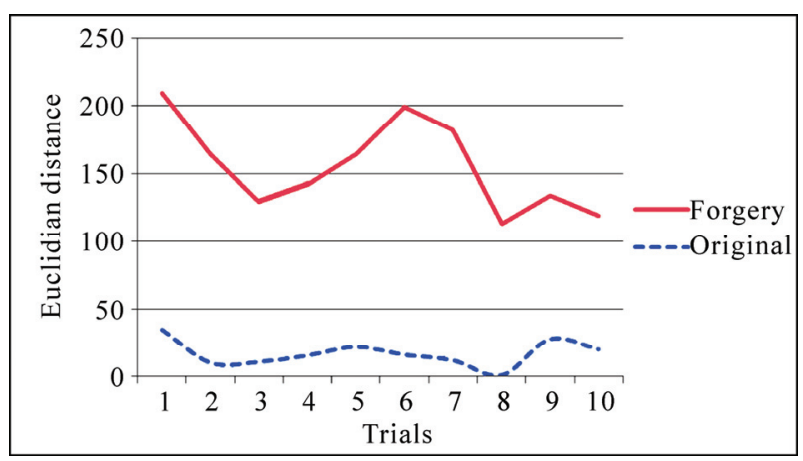

Figure 5. Euclidian distance between genuine signature + ppg factors and forgery.

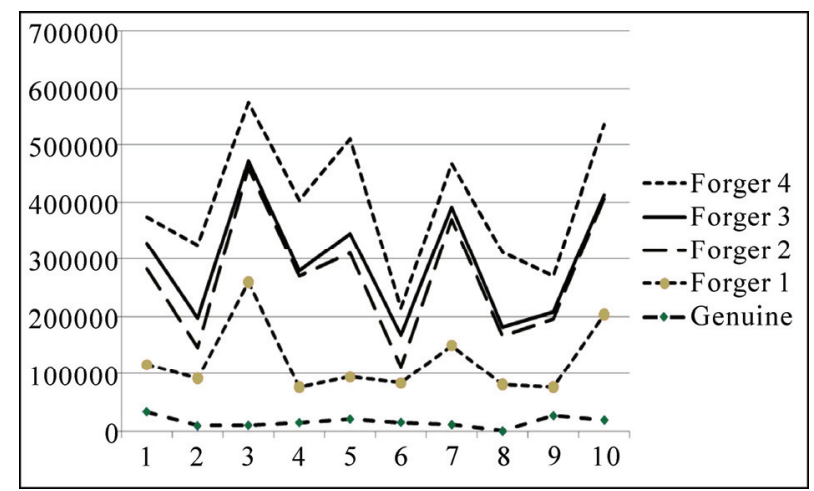

Figure 6. Intra trial variability shown in euclidean distance across ten trials among individual forgers compared with the intra trial variability of genuine features.

Table 1. Intra trial variations in euclidian distance between individual templates to corresponding individual signatures.

\begin{tabular}{|c|c|c|c|c|c|c|c|c|c|c|c|}
\hline & Trial1 & Trial2 & Trial3 & Trial4 & Trial5 & Trial6 & Trial7 & Trial8 & Trial9 & Trial10 & Average Rnd \\
\hline Genuine & 34370 & 9934 & 10759 & 15392 & 21582 & 15866 & 11819 & 875.38 & 27423 & 19917 & 16793 \\
\hline Forger1 & 165410 & 53161 & 198290 & 191950 & 215300 & 28236 & 218790 & 83738 & 118500 & 201180 & 147455 \\
\hline Forger2 & 44223 & 51702 & 12488 & 8906.1 & 33876 & 54862 & 21046 & 15533 & 12128 & 6096.4 & 26086 \\
\hline Forger3 & 46886 & 127180 & 103760 & 123760 & 165250 & 46779 & 75789 & 129740 & 62206 & 124990 & 100634 \\
\hline Forger4 & 82633 & 82805 & 249120 & 62179 & 73780 & 68638 & 138800 & 81469 & 49637 & 184130 & 107319 \\
\hline
\end{tabular}




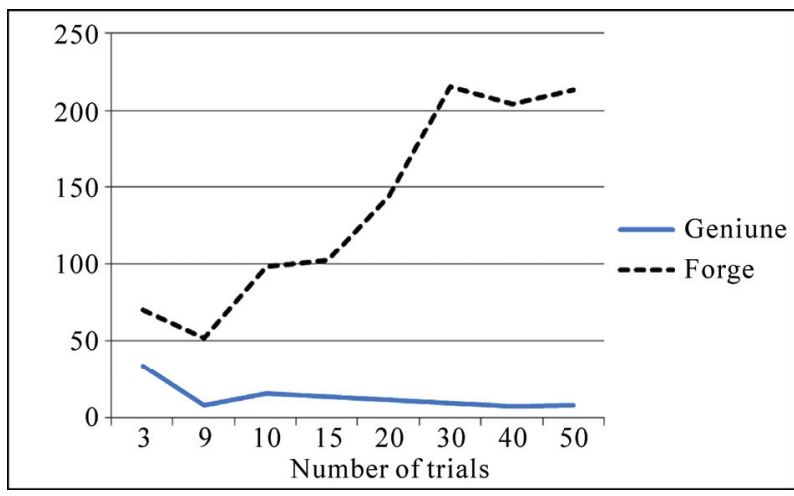

Figure 7. Variability rates against the number of trials.

This wide gap between the intra trial variability shown in Figure 7 reveals that this factor can be considered as an improvement factor in reinforcing the robustness of data glove + PPG based signature verification system.

\section{Conclusions}

The proposed intra trial variability measurement of multi-modal signature + PPG based signature verification system, is found to be reliable in strengthening the identification of genuine subjects of Data glove based signature verification system. The novelty lies in two levels of using PPG factors and its augment to the robustness of the data glove features as well as the counting of Intra trial variability factors against the reference signatures. In feature the data glove may be fabricated as additionally accommodating the PPG sensor to make it easy for everyone. This technique is verified with the present easy modeling of data glove signals using SVD. Two sets of singular vectors produced by SVD are fused as the feature, where the primary set is from data glove with the maximum energy of the signature during the process of signature writing, and the secondary set is derived from the photometric signals extracted by PPG simultaneously during the process has been presented. These selected set of vectors are known as the principle subspace of data glove output matrix $\boldsymbol{A}$ and PPG output matrix $\boldsymbol{B}$ respectively. These principle subspace set are used to model a reinforced signature feature robust against any forge attack.

This research work is a venture to demonstrate the intra trial variability factor enhances the signature verification system that uses the PPG as its combination. This novel system is much potential to offer a sensitive high level security for applications like banking, electronic commerce and legal proceedings, than the existing similar systems. On the other hand this founding strongly supports the reduction in hardware by manufacture data glove integrated with PPG with minimum sensors so that the size of the equipment can be made simple and efficient for handling by a single hand. Since the possibility of reducing the feature size by means of reducing sensor in the data glove as well as reducing timing in the PPG, we can achieve a low cost signature verification system suitable to a common user in common place.

\section{References}

[1] R. Plamondon and S. N. Srihari, "On-Line and Off-Line Handwriting Recognition: A Comprehensive Survey," IEEE Transactions on Pattern Analysis and Machine Intelligence, Vol. 22, No. 1, 2000, pp. 63-84.

[2] S. Rhee, B.-H. Yang and H. H. Asada, "Modelling of Finger Photoplethysmography for Wearable Sensors," Proceedings of 21st Annual International Conference of the IEEE Engineering in Medicine and Biology Society, Atlanta, 1999.

[3] Y. Y. Gu, Y. Zhang and Y. T. Zhang, "A Novel Biometric Approach in Human Verification by Photophlytesmograpic Signals," Proceedings of the 4th IEEE conference on Information Technology Applications in Biomedicine, Birmingham, 2003, pp. 13-14.

[4] A. Samraj, N. G. Noma and S. Sayed, "Quantification of Emotional Features on Phtoplethysomogrpic Wave Forms Using Box Counting Method of Fractal Dimention," Proceedings of the 8th WSEAS International Conference on Circuits, Systems, Electronics, Control \& Signal, Processing (CSECS’09), Puerto De La Cruz, 2009, pp. 24-29.

[5] J. C. Yao, X. D. Sun and Y. B. wan, "A Pilot Study on Using Derivatives of Photop Phlythesomogrpic Signals as Biometric Identifier," Proceedings of 24th Annual International Conference of the IEEE EMBS, 2007, pp. 45764579.

[6] B. Majhi, Y. Santhosh Reddy and D. Prassanna Babu, "Novel Features for off-Line Signature Verification," International Journal of Computers Communication \& Control, Vol. 1, No. 1, 2006, pp. 17-24.

[7] "SVD and Signal Processing: Algorithms, Applications and Architectures," F. Deprettere, Ed., North Holland Publishing Co., Amsterdam, 1989.

[8] N. S. Kamel, S. sayeed and G. A. Ellis, "Glove Based Approach to Online Signature Verification," IEEE Transactions on Pattern Analysis and Machine Intelligence, Vol. 30, No. 5, 2008, pp. 1-5. 